\title{
Research on Initial Construction Model of Sharing Economy Platform under Competitive repertoire

\author{
ZHOU Chun-Yang
}

School of Economics \& Management, Jiangsu University of Science and Technology, Zhenjiang, 212003,China

a1341271082@qq.com

*ZHOU Chun-Yang

Keywords: Sharing economy platform;Competitive action; Competitive repertoire; Complexity; Heterogeneity; Quantity

Abstract: In an increasingly competitive sharing economy market, the platform must quickly build and increase the number of users through competitive action. The sharing economy platform implements a series of competitive strategies for users to aim to improve platform competitiveness. However, few people know how to use these competitive strategies on the competition repertoire level to increase the number of users, and quickly building a platform ecosystem in the early stage.Extending the competitive repertoire theory to the context of the sharing economy market and integrating it with rating in the sharing economy literature. This paper assumes that the platform can integrate these competitive strategies to increase the number of users. This paper also assumes that the impact of these competitive strategies on platform user growth rating is related to application rating. Through the data set, the initial construction behavior of the sharing economy platform is analyzed. The data set is derived from the competition strategy data of the initial (six months) of the domestic sharing economy platform.Finally,the research model framework of the sharing economy platform was constructed.

\section{竞争组合下的共享经济平台初期构建模型研究}

\author{
周春阳 \\ 江苏科技大学经济管理学院，江苏，镇江，212003
}

a1341271082@qq.com

*周春阳

关键词: 共享经济平台; 竞争行为; 竞争组合; 复杂性; 异质性; 数量

\begin{abstract}
摘要：在竞争日益激烈的共享经济市场中，平台必须在初期通过竞争行为来快速构建平台， 提高用户量。共享经济平台对用户实施一系列的竞争策略都是旨在提高平台竞争力。但是， 很少有人知道如何在组合层面上使用这些竞争策略来提高平台的用户量, 从而在初期快速的 构建平台生态系统。将竞争组合理论扩展到共享经济市场背景下，并将其与共享经济文献中 的评分相结合。本文假定平台可以将这些竞争策略作为组合来提高用户量。本文也假定这些 组合的竞争策略对平台用户增长率的影响与应用评分有关。通过数据集对共享经济平台初期 构建行为进行分析, 数据集来源于国内的 120 家共享经济平台初期 (半年) 的竞争策略数据。 最终提出了共享经济平台在初期构建的研究模型框架。
\end{abstract}




\section{1. 引言}

共享经济俨然成为了当下企业创新发展的新方向 ${ }^{[1]}$ 。在不到十年的时间里, 共享经济平台

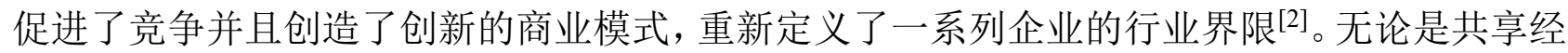
济平台还是研究人员都渴望在拥挤的共享经济市场快速的构建平台, 提高用户量。作为共享 经济的先锋企业: Airbnb 和 Uber 为了在平台初期的时候实现生态系统的快速构建, 提高平台 用户量, 都通过各种竞争行为来与其他平台进行相互对抗 ${ }^{[3]}$ 。以前的文献研究已经在电子商务 和一些共享经济领域确定并检验了一些竞争策略对平台发展构建的影响, 包括定价、投资 ${ }^{[4]}$ 。 所有现存的相关研究都是集中在不同竞争策略对共享经济平台构建的研究, 没有人在竞争组 合层面考虑如何利用它们达到平台的快速构建，提高用户量。

虽然最近美团斥资 37 亿美元收购摩拜这一个体高风险行为能够抢夺各大网站的头条新 闻, 但是许多的共享经济平台往往通过各种各样的动态组合竞争手段来获取优势, 例如降价、 营销活动、新产品发布以及联盟等。百特的竞争对手 Endo Health 在 2010 年发起了比百特多 两倍多的竞争策略举措, 但它将其竞争活动主要集中在两种策略类型中。随着竞争的持续和 时间的推移, 参与竞争对手的一系列复杂竞争行为可以让公司更好地适应不断变化的环境条 件。

战略研究人员称这种竞争组合具有复杂性, 并且在已有的研究中它与企业的绩效有着正 相关的影响 ${ }^{[5]}$ 。除了复杂性这一结构特征之外, 竞争组合还有两个结构特征: 数量, 和异质性 [6]。这些作者将竞争组合理论引入相关平台市场, 并研究这三方面的结构特征对平台绩效的影 响 ${ }^{77]}$ 。然而, 通过对竞争组合的动态模式及其对平台构建的影响进行详细分析, 而不仅仅是简 单和静态的特征描述，对平台的快速构建来说更有用。因此本文的主要研究问题就是共享经 济平台的竞争组合三个结构特征的动态变化与平台初期构建之间的影响关系，从而分析出共 享经济平台初期搭建过程中的模型框架。

先前有关动态竞争战略的研究更多的关注高管团队对竞争行为和平台发展决策的影响, 很少关注有关组织管理和内部结构对竞争行为的影响 ${ }^{[8]}$ 。一方面, 平台的规模可以影响平台竞 争行为的执行速度。另一方面, 评分制度和制度环境在竞争中被视为外生变量研究, 但很少 有人研究其对竞争行为的深入影响。为了进一步的弥补上述研究的不足, 本文将共享经济平 台评分加入到探讨竞争组合结构特征与平台初期构建之间影响关系的研究中。

为了解决这些问题, 本文开发了一个研究模型, 该模型借鉴了动态竞争战略下的竞争组 合理论，结合平台评分制度。本文计划利用平台的数据集来对共享经济平台初期构建过程的 研究模型进行分析, 该数据包括国内 120 家共享经济平台在初期（三年）构建实施的竞争策 略。具体展开了三个方面的研究。首先, 它通过关注动态而不是静态来开发和测试关于竞争 组合 ${ }^{1}$ 结构特征和平台初期构建之间关系的假设。其次, 它将评分制度扩展到共享经济背景下, 进一步探讨应用评分对竞争组合和平台初期构建之间的调节作用。最后, 本文回应了就平台 如何通过研究竞争行为来加强平台构建治理和提升隐藏力量的呼吁。本文首先对竞争战略下 的竞争组合理论和有关平台评分的研究进行简要的文献回顾, 其次是分析平台的竞争策略, 最后在此基础上提出平台初期搭建的研究模型框架。

\section{2. 理论基础}

\section{1 竞争组合理论}

竞争行为被定义为“任何可能获得竞争对手市场份额或者能够降低竞争对手预期业绩 (收

作者简介: 周春阳（1994- ), 女, 江苏海安县人, 研究生。研究方向: 共享经济。E-mail: dbqwanwdn@sina.cn 
入）的可见行为”[9]。关于企业是否以及如何开展竞争行为的分析和计算是动态竞争中最重要 的问题之一。SCP 理论认为企业的任何传导都是在特定的组织结构下实施的, 这样的组织结 构和机制肯定会影响到竞争行为。

在讨论到到针对多个竞争对手的竞争策略的时候, 企业通常会想到学习一些不同的组合。 而竞争组合是竞争动力学文献中的重要研究方向, 竞争组合是指“组织为吸引, 服务和保持顾 客而采取的一系列行动”[10]。它包含“基于特定竞争行为和反应的企业间对抗，企业的战略和 组织背景以及他们的驱动和后果”这几方面的研究 ${ }^{[12]}$ 。竞争组合视角最鲜明的特征在于它关注 整个公司在特定时期内的竞争行为的整个集合（以下称竞争组合）。这种方法允许 研究人员以一种具体的方式丰富而精细地表现竞争战略, 这种观点将战略概念化为决策流中 的一种模式。这种方式与波特的低成本或差异化的一般战略等大多数其他战略概念不同。

竞争组合理论的观点表明它具有三种影响企业发展或绩效的结构特征, 即竞争组合的数 量、竞争组合的复杂性和竞争组合的异质性。竞争组合的数量是指一家公司在某个特定时期 内为了发展或者销售业绩进行的竞争行为总数。竞争组合的复杂性指的是“企业进行的一系列 不间断竞争行为的程度, 包括不同类型的广泛 (非狭險) 的行动范围”。竞争组合的异质性表 示“企业进行的一系列不间断的竞争行为在多大程度上背离了竞争对手的竞争行为”。竞争组 合和企业发展绩效之间的关系已经吸引了瞩目的研究。Ferrier 等人 ${ }^{[11]}$ 研究了在七年期间竞争 组合数量的影响, 竞争组合简单性（复杂性的反义词）以及竞争组合的不相似性（异质性的 同义词）对 40 个传统行业的公司业绩的评估影响。Ndofor ${ }^{[12]}$ 等人认为, 复杂的组合可能会提 高公司业绩, 因为各种各样的行动可能会更充分地利用公司的整个可用资源组合。Ferrier 和 $\mathrm{Lee}^{[13]}$ 在八年的时间里提出了 11 个行业中战略复杂性与企业绩效之间以及战略异质性与企业 绩效之间的 U 型关系。Gnyawali 等人实证检验了三年期间竞争组合数量和竞争组合复杂性对 社交网络平台绩效的影响 (如页面浏览量)。Chua T S 等人研究如何通过应用评分制度区分用 户的兴趣来聚合平台的特定功能。

这些现存的研究使用纵向数据集, 然而, 这些假设是以静态方式呈现和检查, 而不是指 定关系随着时间的推移而变化的变化模式。实际上, 公司需要详细分析竞争组合的动态模式 对企业绩效随着时间推移的影响，而不仅仅是简单和静态的表现。

\section{2共享经济平台的快速构建}

所谓共享经济平台的初期构建阶段就是平台开始获得第一份市场份额或是第一次较大幅 度的用户增长量 (也就是本文的用户增长率) 之前的平台生态系统的快速构建过程。最近势 头正猛的礼橙专车就是诸多共享经济平台中的一个典型例子。现在的礼橙专车已经处于一个 成熟发展的阶段, 它在初期构建平台生态系统的时间在半年左右, 所以这里也是以半年的时 间作为平台的初期构建标准。现如今的很多企业都涉足共享经济平台的各个领域, 企业只有 在初期构建平台的时候实施精准的竞争战略才能快速的打入市场, 抢夺市场份额, 提高注册 用户量, 达到一个初步构建完善成型的共享经济平台生态系统。要想在初期成功的构建共享 经济平台来稳住阵脚以长远的发展下去并不是信手拈来的事情。在前期, 平台拼的是效率, 拼的是资本, 也就是实施准确的平台竞争战略。共享经济平台初期的快速构建在一定程度上 是共享经济平台实施竞争战略的最直接具体的表现。

共享经济平台初期的快速构建需要同时兼备资源和用户两方面的问题, 握紧了资源, 吸 引了用户, 平台才能有立足的根基。然而, 面对一个创新的消费模式, 吸引用户的最大点就 是各大企业争相实施的具体的竞争策略。无论是资源的提供者还是需求者, 利益的驱动远远 高于平台本身带来的便利性。从用户愿意尝试创新的消费模式到逐渐养成消费习惯, 这个过 程会为平台积累很多接受力强的用户以及挖掘大量的潜在用户, 而用户才是一个共享经济平 台得以构建和生存的根本。目前很多企业都纷纷实施不同的竞争策略来抢夺用户。 


\section{3. 研究模型}

在拥挤且竞争激烈的共享经济市场中，平台必须不遗余力地提高平台的用户量，在初期 快速构建平台。一种选择就是实施大量的竞争策略来增加自身的竞争力。这些竞争策略包括 提供降价和销售激励的与定价相关的功能; 营销相关职能; 与支付渠道相关的支付优惠功能。 借鉴竞争组合理论文献, 本文将共享经济市场平台对这些平台的竞争策略的使用概念化为他 们的竞争行为。因此，我们将共享经济市场平台的竞争组合定义为在一段时间内，由平台部 署的为了提高平台用户量实现平台构建而实施的一整套竞争策略。具体的竞争策略如表 1 所 示。

表 1 共享经济平台竞争策略分类汇总

\begin{tabular}{cc}
\hline 竞争策略 & 具体竞争行为 \\
\hline 定价 & 订单交易、按时计费、押金、包时付费 \\
营销活动 & 线上推广、线下推广、线上营销、线下活动 \\
运营优惠 & 直接优惠、间接优惠 \\
\hline
\end{tabular}

依据本文研究的主要问题: 共享经济平台竞争组合与平台初期构建之间的影响关系以及 平台作为 App 在应用中的评分如何进一步的调节两者之间的关系。本文研究模型如图 1 所示。

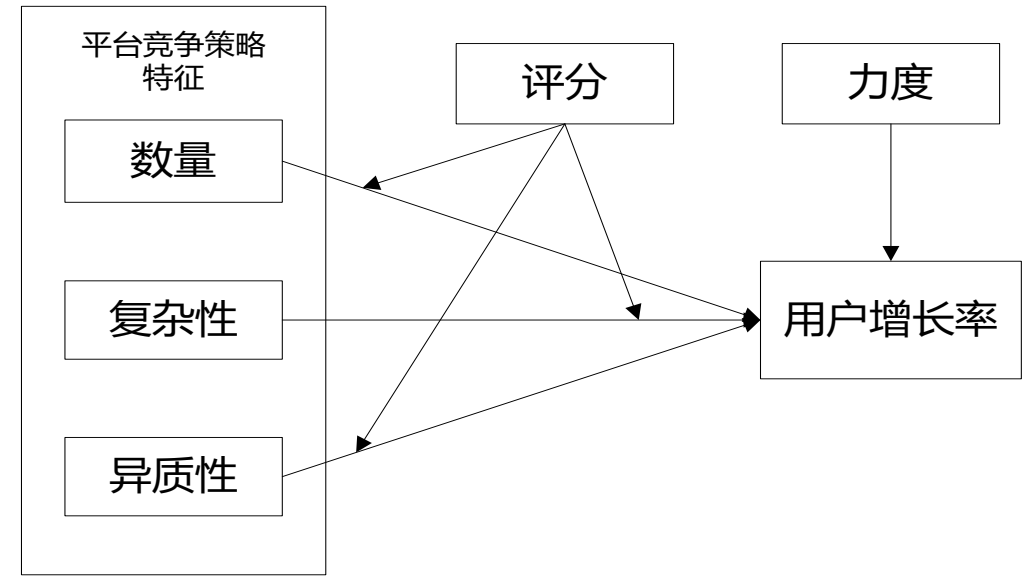

图 1 研究模型

\section{4. 结论}

为了研究共享经济平台竞争组合对平台初期快速构建的影响, 本文基于国内 120 家共享 经济平台的竞争策略数据, 从竞争组合数量、复杂性、异质性三方面的结构特征讨论对平台 初期构建模型的影响, 同时加入平台的应用评分作为调节变量来调节竞争组合数量、复杂性、 异质性与平台初期构建之间的关系, 最终得出了竞争组合影响下的共享经济平台初期构建研 究模型框架。

\section{致谢}

基金项目: 江苏省普通高校研究生科研计划项目(KYCX18_2302)

\section{Reference}

[1] Mocker M, Fonstad N O. How AUDI AG is driving toward the sharing economy[J]. Mis Quarterly Executive, 2017, 16(4):279-293.

[2] Constantiou I D, Marton A, Tuunainen V K. Four Models of Sharing Economy Platforms[J]. Mis 
Quarterly Executive, 2017.

[3] Zervas G, Proserpio D, Byers J. The Rise of the Sharing Economy: Estimating the Impact of Airbnb on the Hotel Industry[J]. Social Science Electronic Publishing, 2016.

[4] Walter J. Ferrier, Ken G. Smith, Curtis M. Grimm. The Role of Competitive Action in Market Share Erosion and Industry Dethronement: A Study of Industry Leaders and Challengers[J]. The Academy of Management Journal, 1999, 42(4):372-388.

[5] Yat-Hung Chiang, Bo-Sin Tang, Francis K. W. Wong. Volume building as competitive strategy[J]. Construction Management \& Economics, 2008, 26(2):161-176.

[6] Angappa Gunasekaran, Kee-hung Lai, T.C. Edwin Cheng. Responsive supply chain: A competitive strategy in a networked economy 话[J]. Omega, 2008, 36(4):549-564.

[7] Shang X, Chen H. Competitive Strategy and Regulations of Platform Firms from the Perspective of Bilateral Market[J]. Industrial Economics Research, 2009, 10(6):2461-2472.

[8] Healy P M, Palepu K G. Information asymmetry, corporate disclosure, and the capital markets: A review of the empirical disclosure literature ${ }_{2}[\mathrm{~J}]$. Journal of Accounting \& Economics, 2004, 31(1-3):405-440.

[9] S. Venkataraman, Ming-Jer Chen, Macmillan I C. Anticipating Reactions: Factors that Shape Competitor Responses[J]. Social Science Electronic Publishing, 1997.

[10]John A. Mathews. Competitive dynamics and economic learning: an extended resource-based view[J]. Industrial and Corporate Change, 2003, 12(1):115-145.

[11]Walter J. Ferrier, Ken G. Smith, Curtis M. Grimm. The Role of Competitive Action in Market Share Erosion and Industry Dethronement: A Study of Industry Leaders and Challengers[J]. The Academy of Management Journal, 1999, 42(4):372-388.

[12]Ndofor H A, Sirmon D G, He X. Firm resources, competitive actions and performance: investigating a mediated model with evidence from the in-vitro diagnostics industry[J]. Strategic Management Journal, 2011, 32(6):640-657.

[13]Ferrier W J, Lee H. Strategic Aggressiveness, Variation, And Surprise: How The Sequential Pattern Of Competitive Rivalry Influences Stock Market Returns[J]. Journal of Managerial Issues, 2002, 14(2):162-180. 